\title{
Grid Computing Environment Using Ontology Based Service
}

\author{
Ana Marilza Pernas ${ }^{1}$ and Mario Dantas ${ }^{2}$ \\ 1 Department of Informatics, Federal University of Pelotas, \\ 99010-900 Pelotas, Brazil \\ ampernas@ufpel.edu.br \\ 2 Department of Informatics and Statistics, Federal University of Santa Catarina, \\ 88040-900 Florianopolis, Brazil \\ mario@inf.ufsc.br
}

\begin{abstract}
Grid computing environments can share resources and services in a large-scale. These environments are being considered as an effective solution for many organizations to execute distributed applications to obtain high level of performance and availability. However, the use of a grid environment can be a complex task for an ordinary user, demanding a previous knowledge of the access requirements from a virtual organization. In order to improve the search of resources and its selection, in this paper we propose the use of ontology as alternative approach to help the use of grid services. This paradigm can provide a description of available resources, leading users to desire operations and describing resources syntax and semantics that can form a common domain vocabulary. Our experimental result indicates that our proposal allows a better comprehension about available resources.
\end{abstract}

\section{Introduction}

A grid environment can be understood as a set of services, provided by institutions to be used from another. Its architecture may be viewed as service-oriented [1], where two entities have a special importance: the producer and the consumer of the service. In this vision, owners offer services to be used, according to some restrictions that must be satisfied before given access permission to consumer. Because of these restrictions and rules of sharing, the service oriented grid architecture requires a scheme to support the interoperability between applications from the users and a high level of access transparency for resources.

Considering a service oriented approach, in this article we propose the use of ontology for the description of available resources in a grid computing environment. The motivation of this research is based on the clear advantages in using ontology, as some projects have shown (e.g. $[2,3,4])$, to have a common domain of concepts shared among ordinary users. The use of ontology for the semantics description of a vocabulary can provide a clear understanding of characteristics and properties of classes and relations. 
The paper is organized as follows. The development of the proposed ontology based service is described in section 2 . In section 3, we present some related works. In section 4 we present our conclusions and future work.

\section{Ontology for Grid Resources Description}

In this section we present the ontology created. In addition to the axioms, other two structures help the ontology in the description of resources:

- Metadata reflects the information related to a data. In this research, metadata stores information about computational resources;

- Semantics View - stores information related to the present state of a resource. Thus, when a request comes, these structure returns information about that moment.

Even not commonly used in other ontologies, these structures can improve the ontology action, returning answers more quickly. The ontology was implemented using the OWL Full language [5], and was edited using Protégé-2000 editor [6].

The ontology works in the directory of a grid configuration. Fig. 1 (modified from [7]) shows that consumers' queries come to the ontology. To obtain information about computational resources, metadata receives information from catalogs and data files, while semantic views communicates with the Metacomputing Directory Service (MDS), which provides a distributed access to the grid structure and information related to system components.

\subsection{Ontology Development}

To probe how to describe the concepts related to grid computational resources, we search for the vocabulary utilized by the following projects: NPACI [8], ESG [9]; NASA's Information Power Grid [10] and the Distributed ASCI Supercomputer Project 2 [11]. After our search we create the documentation required to

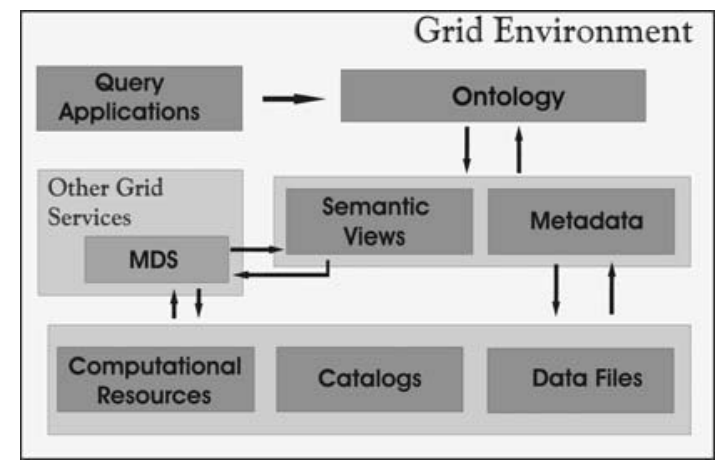

Fig. 1. Grid architecture using the ontology approach 


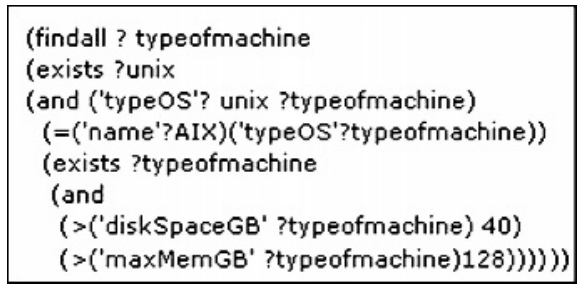

Fig. 2. Axiom to access restriction

build the ontology, witch consists of all concepts, described in a formally way and mapped in classes and instances, that will form the ontology.

Our next task was to reproduce this documentation to the OWL language, using the Protégé editor. In the editor, we also created axioms, employing the PAL (Protégé Axiom-Language). Fig. 2 shows an axiom to access restriction, where is only possible to occur if the operating system from the resource is AIX, disk space greater then 40 GB and memory greater then 128 GB. Metadata were also created using Protégé and OWL language.

\subsection{Ontology Based Service}

To develop the grid service we created an application, using Java Language, to allow the service to interact with the ontology. The application was designed with three modules. The first module provides a list of all classes and instances defined in the ontology. In the second module, consumers can probe for metadata from any class listed by the first module. The third module allows a search of any computational resource, where a consumer can visualize the entire configuration. The service was defined using the Globus toolkit [12] and it is characterized by the application executing in a grid configuration. The application was configured to accept incoming connections from consumers of a grid. In this new environment, computational resources are presented using a more clear description, as we verified with users from our Federal University configuration.

\section{Related Work}

Concerning to the resources selection in a grid environment, is possible to find some research works (e.g. $[3,13]$ ), where authors use the ontology to help the use of a grid environment. Some research works are related to apply the ontology in existing grid environments. One of theses efforts is the Semantic Grid [1], a grid infrastructure which has the goal to support applications related to e-Science.

\section{Conclusion and Future Work}

In this paper we presented a research work to provide access transparency to users of grid configurations. Our approach was based on ontology. We first presented some concepts of ontology and service oriented in grid configurations. 
The environment of our prototype was described starting with the methodology used, followed by some characteristics of the development and finally how the ontology base service works. The system has proved to be an efficient and friendly approach to provide grid resources to consumers. As a future research work we are planning to enhance the system to allow some dynamic changes, such as metadata or inclusion, on the application. Other work is to create an ontology to agriculture field and use the application on more wide and complex grid environment.

\section{References}

1. De Roure, D., Jennings, N.R., Shadbolt N.R.: The Semantic Grid: A Future eScience Infrastructure. In: Grid Computing: Making The Global Infrastructure a Reality, F. Berman, A.J.G. Hey and G. Fox (eds), Southern Gate, Chinchester, England: John Wiley Sons, 1080p. (2003) 437-470.

2. Pernas, A. M.: Ontologias Aplicadas a Descrio de Recursos em Grids Computacionais. Dissertation (Master Degree), Federal University of Santa Catarina, Florianpolis, Brazil (2004).

3. Tangmunarunkit, H., Decker, S., Kesselman, C.: Ontology-based Resource Matching - The Grid meets the Semantic Web. 1th Workshop on Semantics in Peer-toPeer and Grid Computing (SemPGrid) at the 12th International World Wide Web Conference, Budapest, May (2003).

4. Heine, F., Hovestadt, M., Kao, O.: Towards Ontology-Driven P2P Grid Resource Discovery. 5th IEEE/ACM International Workshop on Grid Computing, Pittsburgh, USA,November(2004).

5. McGuinness, D., Van Harmelen, F.: Web Ontology Language Overview. (2004). Available online http://www.w3.org/TR/owl-features/.

6. Noy, N., Fergerson, R., Musen, M.: The knowledge model of Protege-2000: Combining interoperability and flexibility. 12th Int. Conference on Knowledge Engineering and Knowledge Management(EKAW), French Riviera, October (2000) 2-6.

7. Goble, C., De Roure, D.: Semantic Web and Grid Computing. September (2002). Available online: http://www.semanticgrid.org/documents/swgc/.

8. NPACI-National Partnership for Advanced Computational Infrastructure: Partnership Report. (2000). Available online: http://www.npaci.edu/.

9. Foster, I., Middleton, D., Williams, D.: The Earth System Grid II: Turning Climate Model Datasets into Community Resources. January (2003). Available online: https://www.earthsystemgrid.org/about/docs/ESGOverviewSciDACPINapa_v8. doc.

10. IPG, Information Power Grid - Nasa's Computing and Data Grid: What is the IPG? October (2002). Available online: http://www.ipg.nasa.gov/aboutipg/ what.html.

11. Verstoep, K.: The Distributed ASCI Supercomputer 2 (DAS-2). May (2000). Available online: http://www.cs.vu.nl/das2/.

12. Foster I., Kesselman, C.: The Globus Project: a Status Report. In: Proc. of 7th Heterogeneous Computing Workshop (HCW 98), March (1998) 4-18.

13. Pouchard, L. et.al.: An Ontology for Scientific Information in a Grid Environment: the Earth System Grid. In: Proc. of the 3th IEEE/ACM International Symposium on Cluster Computing and the Grid, Japan, Tokyo, May (2003) p. 626. 